\title{
Long bone failure after intraosseous regional perfusion
}

\author{
V. Slack-Smith ${ }^{1}$, H.M.S. Davies ${ }^{2}$ and B.J. Hilbert ${ }^{1^{*}}$
}

${ }^{1}$ Veterinary Clinical Centre, Charles Sturt University, Wagga Wagga, New South Wales 2678, and

Australia and 2 Department of Veterinary Biosciences, University of Melbourne, Parkville, Victoria 3010, Australia.

*Corresponding author email: bhilbert@csu.edu.au

Current address for Dr Slack-Smith: 1 Gladevale Street, Julia Creek, Queensland 4823, Australia.

Keywords: horse; sepsis; antimicrobials; intraosseous regional perfusion; fracture

\section{Summary}

A horse that had sustained a penetrating injury to the proximal and medial aspect of the left fore MC3 was presented to the Charles Sturt University Veterinary Clinical Centre (CSUVCC). The horse had initially been treated with bandaging and systemic antimicrobials by the referring veterinarian but had failed to respond. Radiographs of the limb made at CSUVCC revealed bone lysis and a palisading new bone reaction on the medial splint bone that were thought to be characteristic of infection (Fig 1). As the associated soft tissue swelling extended above and below the carpus and therefore precluded intravenous regional perfusion (IVRP), a decision was made to use intraosseous regional perfusion (IORLP) to deliver antimicrobials to the limb. The horse was sedated with detomidine and butorphenol and an area of skin and subcutaneous tissue on the lateral diaphysis of MC3 was desensitised using an infiltration of $5 \mathrm{~mL}$ of lignocaine hydrochloride. A $4.5 \mathrm{~mm}$ bone portal was made in the lateral diaphysis of the left fore MC3 using the technique described by Scheuch et al. (2002). After a rubber tourniquet was applied to the limb above the carpus, one third of the calculated systemic dose of gentamicin sulphate was diluted with $30 \mathrm{~mL}$ of normal saline and injected slowly into the medullary cavity via a cannulated screw and a short i.v. extension tube. The tourniquet was removed after 30 minutes and the leg was supported with a padded bandage. The horse was maintained on systemic antimicrobials for 7 days and using the same portal, the IORLP procedure was repeated 3 times at 48 -hour intervals.

This is the author manuscript accepted for publication and has undergone full peer review but has not been through the copyediting, typesetting, pagination and proofreading process, which may lead to differences between this version and the Version of Record. Please cite this article as doi: $10.1111 /$ EVE.13172

This article is protected by copyright. All rights reserved 
Approximately 13 weeks after the IORLP, the horse was re-presented to the CSUVCC for further assessment of lameness and a swelling centred over the LF cannon bone in the vicinity of the original IORLP access portal. Radiographs revealed a longitudinal fracture of MC3 (Fig 2) which appeared to have propagated from the drill hole and the swelling was identified as a bony callus. Fortunately, the horse had survived this fracture event and had undergone a healing process that stabilised the bone. At the time of writing, the horse is sound and the healing process has continued.

\section{Key Points}

- Intraosseous regional limb perfusion is a recognised method of delivering high concentrations of antimicrobials to infected tissues.

- Long bone fracture in the horse as a complication of this technique has not been reported.

- More work is needed to determine the safest site for making an IORLP access portal in MC3 and other equine bones.

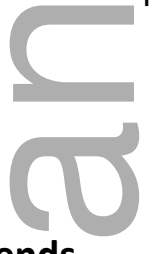

Figure legends

Fig 1: DMPLO radiograph showing bone lysis (arrow head) and the palisading bone reaction on MC2 (block arrow).

Fig 2: LM radiograph showing the bone portal (block arrow) and the associated fracture lines (arrow heads).

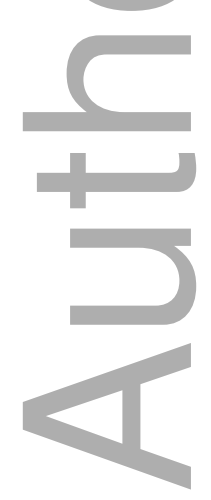


Article type : Case Report

6

7

Long bone failure after intraosseous regional perfusion

9 V. Slack-Smith ${ }^{1}$, H.M.S. Davies ${ }^{2}$ and B.J. Hilbert ${ }^{{ }^{*}}$

$10{ }^{1}$ Veterinary Clinical Centre, Charles Sturt University, Wagga Wagga, New South Wales 2678,

11 Australia and ${ }^{2}$ Department of Veterinary Biosciences, University of Melbourne, Parkville, Victoria 123010 , Australia.

*Corresponding author email: bhilbert@csu.edu.au

Current address for Dr Slack-Smith: 1 Gladevale Street, Julia Creek, Queensland 4823, Australia.

Keywords: horse; sepsis; antimicrobials; intraosseous regional perfusion; fracture

\section{Summary}

A five-year-old Australian Stock Horse gelding was initially referred to the Charles Sturt University Veterinary Clinical Centre (CSUVCC) for assessment of a penetrating laceration over the medial splint bone of the left foreleg. Clinical examination failed to reveal a communication with a synovial structure but radiographs showed a palisading bone reaction on the proximal aspect of the medial splint bone which was thought to be a characteristic of infection. Soft tissue swelling precluded access to a peripheral vein so a decision was made to use intraosseous regional limb perfusion. This was achieved through an access portal in the lateral diaphysis of MC3. Subsequently the horse developed lameness and further investigation revealed a non-displaced longitudinal fracture of MC3 propagating from the intraosseous regional perfusion access portal. 
Intraosseous (IORLP) and intravenous regional limb perfusion (IVRLP) have become popular techniques for delivering high concentrations of antimicrobials to target tissues (Whitehair et al. 1992; Butt et al. 2001; Rubio-Martinez and Cruz 2006; Alkabes et al. 2011; Errico et al. 2012; RubioMartinez et al. 2012). The hypothesis is that delivery of high concentrations of concentrationdependant antimicrobials to infected tissues at or above the minimum inhibitory concentration would be more likely to eliminate bacteria. It has been shown that regional limb perfusion in both rabbits and humans will achieve high concentrations of antimicrobials even in poorly perfused and chronically infected tissues (Finsterbush et al. 1970; Finsterbush and Weinberg 1972). The techniques involve delivery of the perfusate (antimicrobial) directly into the limb through an intravenous or intraosseous portal and distal to an occlusive tourniquet. Thus achieving a concentration and pressure gradient across the intravascular and extravascular compartments (Rubio-Martinez and Cruz 2006). Intraosseous regional limb perfusion is a more invasive technique which involves the creation of a portal into the medullary cavity in the region of the septic site and distal to the tourniquet. The technique is particularly useful when there is damage to and swelling of soft tissues and access to a peripheral vein is challenging. After drilling a unicortical hole, a cannulated, self-tapping bone screw is used as a conduit for the antimicrobial. The same hole can be used for injection on consecutive days, thus avoiding damage to peripheral veins. In 2004, Mattson et al. described an intraosseous technique for the delivery of gentamicin into the distal metacarpus in standing, sedated horses. He used a $4.0 \mathrm{~mm}$ drill and a $5.5 \mathrm{~mm}$ tap for use with a $5.5 \mathrm{~mm} \times 20 \mathrm{~mm}$ cannulated bone screw. The portal was made in the distal metacarpus on the dorsolateral cortex 1 $\mathrm{cm}$ lateral to the common digital extensor tendon. From this work the authors concluded that IORLP of gentamicin using the distal aspect of the limb should be considered for the treatment of orthopaedic infections in horses. Keys et al. (2006) in an experimental study on cadaveric limbs showed that after IORLP the pattern of distribution to the peripheral vessels was in a centrifugal direction from the medullary cavity. However, they showed that this distribution included veins and arteries and that these results supported the use of IORLP to deliver medications to the tissues of the distal forelimb. in a standing, sedated horse

\section{Clinical History}


In 2016, a horse that had sustained a penetrating injury to the proximal and medial aspect of the left fore MC3 was presented to the Charles Sturt University Veterinary Clinical Centre (CSUVCC). The horse had initially been treated with bandaging and systemic antimicrobials by the referring veterinarian, but had failed to respond. The horse was (AAEP) Grade 3/4 lame in the affected limb and palpation of the swelling over the medial splint bone elicited a marked pain response. Radiographs of the limb made at CSUVCC revealed bone lysis and a palisading new bone reaction on the medial splint bone that were thought to be characteristic of infection (Fig 1). As the associated soft tissue swelling extended above and below the carpus and therefore precluded intravenous regional perfusion (IVRLP), a decision was made to use IORLP to deliver antimicrobials to the limb. The horse was sedated with detomidine (Dozadine $0.01 \mathrm{mg} / \mathrm{kg}$ i.v.) ${ }^{\mathrm{a}}$ and butorphanol (Butorgesic $0.02 \mathrm{mg} / \mathrm{kg}$ i.v. $)^{b}$ and an area of skin and subcutaneous tissue on the lateral diaphysis of MC3 was desensitised using an infiltration of $5 \mathrm{~mL}$ of lignocaine hydrochloride (Lignocaine $2020 \mathrm{mg} / \mathrm{mL})^{\text {b }}$. A $4.5 \mathrm{~mm}$ lateral bone portal was made in the diaphysis of the left fore MC3 using the technique described by Scheuch et al. (2002). After a rubber tourniquet was applied to the limb above the carpus, one third of the calculated systemic dose of gentamicin sulphate (Gentam $1006.6 \mathrm{mg} / \mathrm{kg}$ bwt i.v.) ${ }^{b}$ was diluted with $30 \mathrm{~mL}$ of $0.9 \%$ sodium chloride solution and injected slowly into the medullary cavity via the $4.5 \mathrm{~mm}$ cannulated screw ${ }^{d}$ and a short i.v. extension tube (Discofix C) ${ }^{c}$. The tourniquet was removed after 30 minutes and the leg was supported with a padded bandage. The horse was maintained on oxytetracycline ${ }^{e}$ (Engermycin $10 \mathrm{mg} / \mathrm{kg}$ i.v. q12h) and phenylbutazone ${ }^{f}$ (Bute Paste $2.2 \mathrm{mg} / \mathrm{kg}$ PO q12h) for 7 days and using the same portal, the IORLP procedure was repeated 3 times at 48-hour intervals. The skin incision for the IORLP was covered with a bandage and allowed to heal by second intention. The lameness gradually improved during the period of hospitalisation and the phenylbutazone was discontinued.

Approximately 13 weeks after the IORLP, the horse was re-presented to the CSUVCC for further assessment of lameness and a swelling centred over the LF cannon bone in the vicinity of the drill hole. Radiographs revealed a longitudinal fracture of MC3 (Fig 2) which appeared to have propagated from the drill hole and the swelling was identified as a bony callus (Fig 3). Fortunately the horse had survived this fracture event and had undergone a healing process that stabilised the bone. At the time of writing, the horse is sound and the healing process has continued.

\section{Discussion}


This report describes a potentially catastrophic complication of IORLP. The horse developed a fracture at the site of the IORLP at some time after the procedure. The only thing that alerted us to the presence of a complication was that the owner reported a new swelling and lameness after the initial treatment period. Although Mattson et al. (2004) alluded to the possibility of an iatrogenic fracture occurring through an IORLP bone portal, to our knowledge such a long bone fracture in the horse has not previously been reported.

In this case, for safety reasons and ease of access in the standing horse, the IORLP access hole was made on the lateral side of the diaphysis of MC3. On reviewing the case, the question was asked if choosing this particular site predisposed the MC3 to failure when placed under stress. In one study that examined the effects of hole diameter on the torsional mechanical properties of isolated equine cadaveric third metacarpal bones, it was found that the presence of lateral to medial 3/16 inch or $5 / 16$ inch bicortical holes reduced all torsional structural properties (except stiffness) of the bone. Failure torque and post yield properties were most dramatically reduced. The authors hypothesised that these changes may prohibit affected horses from sensing pre-failure damage, enhancing the likelihood of catastrophic failure when bone torsional load exceeds the yield point (Selzer et al. 1996).

The horse in this report was given free access to a small pasture after the IORLP procedure, so the exercise was uncontrolled but limited in its intensity. In a treadmill exercise study, Davies (2005) recorded the timing and distribution of surface strains from strain gauges distributed around the periphery of the mid MC3 cortex of a 3-year-old Thoroughbred racehorse. That study showed that peak compressive strains on the dorsal cortex of MC3 occurred in the first third of stance and were probably related to deceleration of the $\operatorname{limb}$ as these strains were directly proportional to exercise speed. Whereas, strains on the medial cortex were thought to be directly related to support of the body weight. The strains on the lateral cortex occurred at variable times through stance and showed a much larger variation than strains recorded on the other cortices. The lateral strains may be associated with the maintenance of balance as well as the support of body weight. The lateral cortex was not loaded significantly during exercise in a straight line on a flat treadmill surface in that study, but exercise in circles significantly loads the lateral cortex of the inside limb in both compression and tension (Davies and Merritt 2004). This inconsistent pattern of loading of the lateral cortex and the increase in both tensile and compressive strains might help to explain the lateral condylar fractures that occur in racehorses on the turns, and may have been significant in our case.

While these studies are helpful in explaining the strains imparted to MC3 in normal horses, more work needs to be done to determine the safest site for making an IORP access portal in horses with orthopaedic infections. As can be seen in Fig 3, the lateral cortex of MC3 in this case was much 
narrower than the medial cortex which suggests that this horse may not have had sufficient residual strength in that region of bone to withstand sharp turns towards that limb following the surgery. It may be preferable to consider drilling into the medial cortex given that it is apparently more consistently loaded than the lateral cortex (Davies and Merritt 2004). Changes in bone density and loss of structural integrity associated with disease will have some effect on the outcome, but in most cases these will be unknowns. However, any evidence may help surgeons decide on the safest protocol, and it may also enable them to make better post-operative confinement and exercise plans.

Authors' declaration of interests

No conflicts of interest have been declared.

\section{Ethical animal research}

Research ethics committee oversight was not required. The owners gave their verbal consent to use the animal radiographs for educational purposes.

\section{Source of funding}

140 None.

\section{Authorship}

V. Slack-Smith and B. Hilbert undertook the clinical work. H.M.S. Davies provided expert biomechanical interpretation. All authors contributed to the production of the manuscript and approved the final version.

Manufacturers' addresses

${ }^{1}$ Virbac Australia PTY LIMITED, Milperra, NSW 2214, Australia.

$148{ }^{2}$ Ilium, Troy Laboratories PTY LIMITED, Glendinning, NSW 2861, Australia.

${ }^{3}$ B Braun, Australia, Bella Vista, NSW 2153, Australia.

$150{ }^{4}$ The Edge Equine, Bendigo, Victoria 3552, Australia.

$151{ }^{5}$ Intervet AUSTRALIA, PTY LTD, Bendigo East, Victoria 3550, Australia. 
1. Alkabes, S.B., Adams, S.B., Moore, G.E. and Alkabes, K.C. (2011) Comparison of two tourniquets and determination of amikacin sulfate concentrations after metacarpophalangeal joint lavage performed simultaneously with intravenous regional perfusion. Am. J. Vet. Res. 72, 613-619.

2. Butt, T.D., Bailey, J.V., Dowling, P.M. and Fretz, P.B. (2001) Comparison of two techniques for regional antibiotic delivery to the equine forelimb: intraosseous vs. intravenous perfusion. Can. Vet. J. 42, 617-622.

3. Davies, H.M.S. (2005) The timing and distribution of strains around the surface of the third metacarpal bone during treadmill exercise in one Thoroughbred racehorse. Aust. Vet. J. 83, 157-163.

4. Davies, H.M.S., and Merritt, J.S. (2004) Surface strains around the midshaft of the third metacarpal bone during turning. Equine Vet. J. 36, 689-692.

5. Errico, J.A., Trumble, T.N., Bueno, A.C., Davis, J.L. and Brown, M.P. (2012) Comparison of two indirect techniques for local delivery of a high dose of an antimicrobial in the distal portion of forelimbs of horses. Am. J. Vet. Res. 69, 334-342.

6. Finsterbush, A., Argaman, M. and Sacks, T. (1970) Bone and joint perfusion with antibiotics in the treatment of experimental staphylococcal infection in rabbits. J. Bone Joint Surg. Am. 52, 1424-1434.

7. Finsterbush, A. and Weinberg, H. (1972) Venous perfusion of the limb with antibiotics for osteomyelitis and other chronic infections. J. Bone Joint Surg. Am. 54, 1227-1234.

8. Kearney, C. (2015) What is the most effective way to perform intravenous regional perfusion. Equine Vet. Educ. 27, 442-443.

9. Keys, G.J., Berry, D.B., Pleasant, R.S., Jones, J.C. and Freeman, L.E. (2006) Vascular distribution of contrast medium during intraosseous regional perfusion of the distal portion of the equine forelimb. Am. J. Vet. Res. 67, 1445-1452.

10. Mattson, S., Boure, L., Pearce, S., Hurtig, M., Burger, J. and Black, W. (2004) Intraosseous gentamicin perfusion of the distal metacarpus in standing horses. Vet. Surg. 33, 180-186.

11. Rubio-Martinez, L.M. and Cruz, A.M. (2006) Antimicrobial regional perfusion in horses. J. Am. Vet. Med. Assoc. 228, 706-712.

This article is protected by copyright. All rights reserved 
12. Rubio-Martinez, L.M., Elmas, C.R., Black, B. and Monteith, G. (2012) Clinical use of antimicrobial regional limb perfusion in horses: 174 cases (1999-2009). J. Am. Vet Med. Assoc. 241, 1650-1658.

13. Scheuch, B.C., Van Hoogmoed L.M., Wilson W.D., Snyder J.R., McDonald M.H., Watson Z.E. and Steffey, E.P. (2002) Comparison of intraosseous or intravenous infusion for delivery of amikacin sulfate to the tibiotarsal joint of horses. Am. J. Vet. Res. 63, 374-380.

14. Selzer, K.L., Stover, S.M., Taylor, K.T. and Willits, N.H. (1996) The effect of hole diameter on the torsional mechanical properties of the equine third metacarpal bone. Vet. Surg. 25, 371375 .

15. Whitehair, K.J., Blevins, W.E., Fessler, J.F., Van Sickle, D.C., White, M.R. and Bill, R.P. (1992) Regional perfusion of the equine carpus for antibiotic delivery. Vet. Surg. 21, 279-285.

\section{Figure legends}

Fig 1: DMPLO radiograph showing bone lysis (arrow head) and the palisading bone reaction on MC2 (block arrow).

Fig 2: LM radiograph showing the bone portal (block arrow) and the associated fracture lines (arrow heads).

Fig 3: DP radiograph showing the portal (block arrow) and the new bone reaction on MC3 (arrow head).

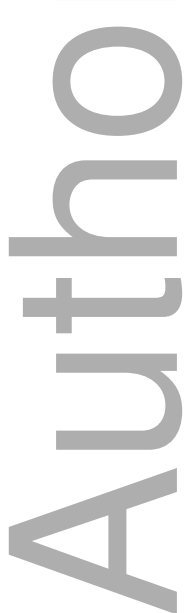

This article is protected by copyright. All rights reserved 


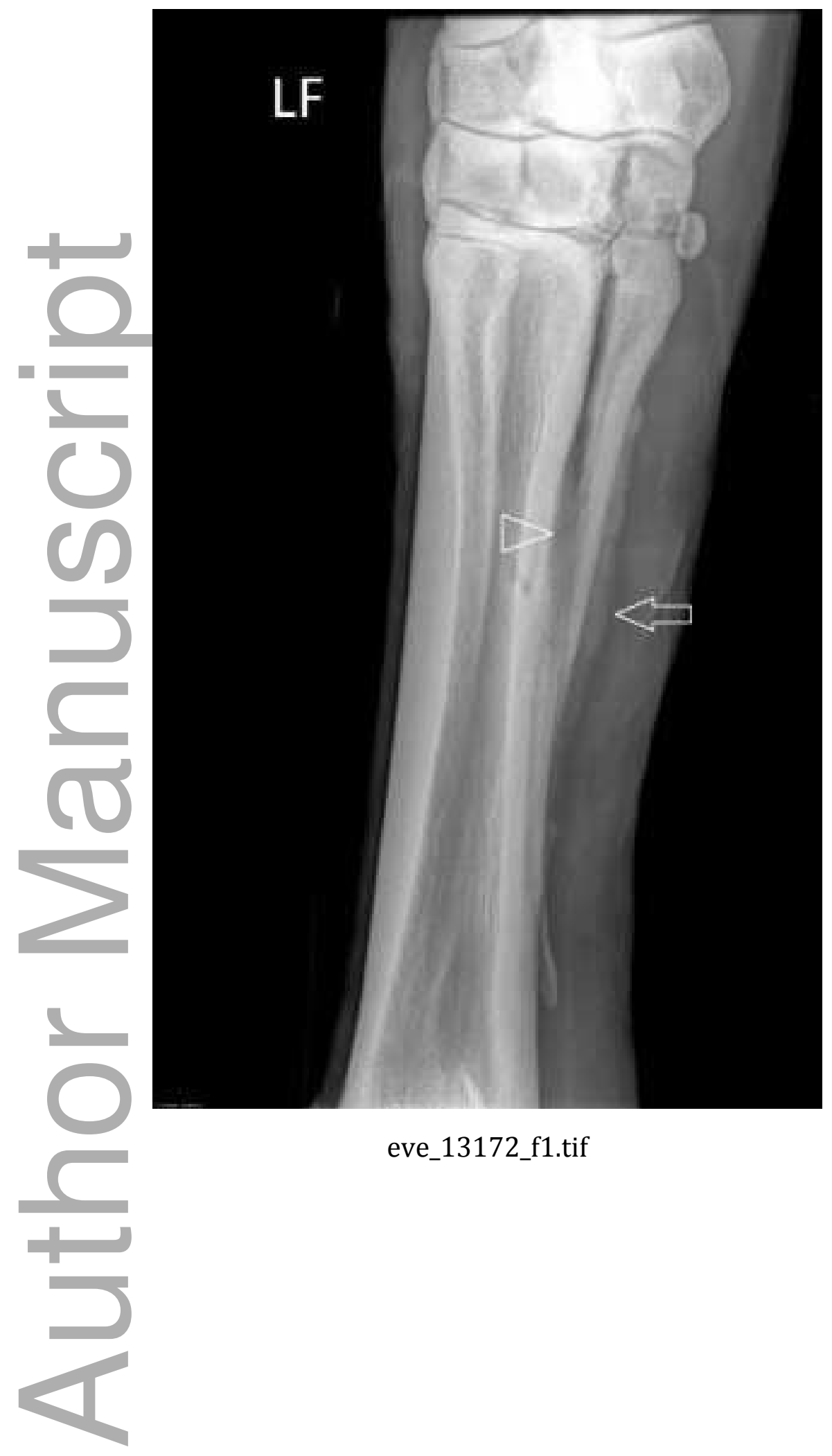

This article is protected by copyright. All rights reserved 


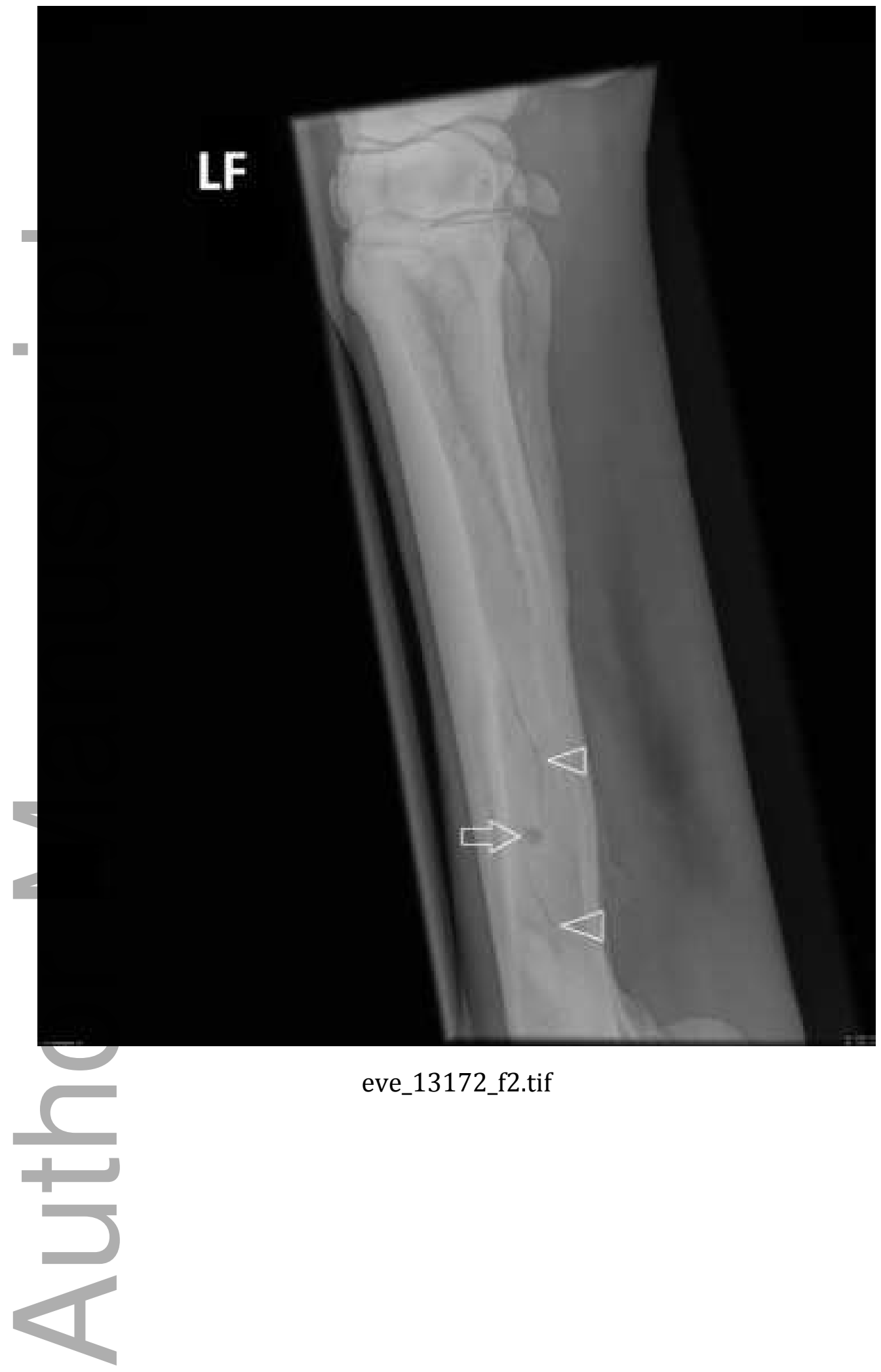

This article is protected by copyright. All rights reserved 


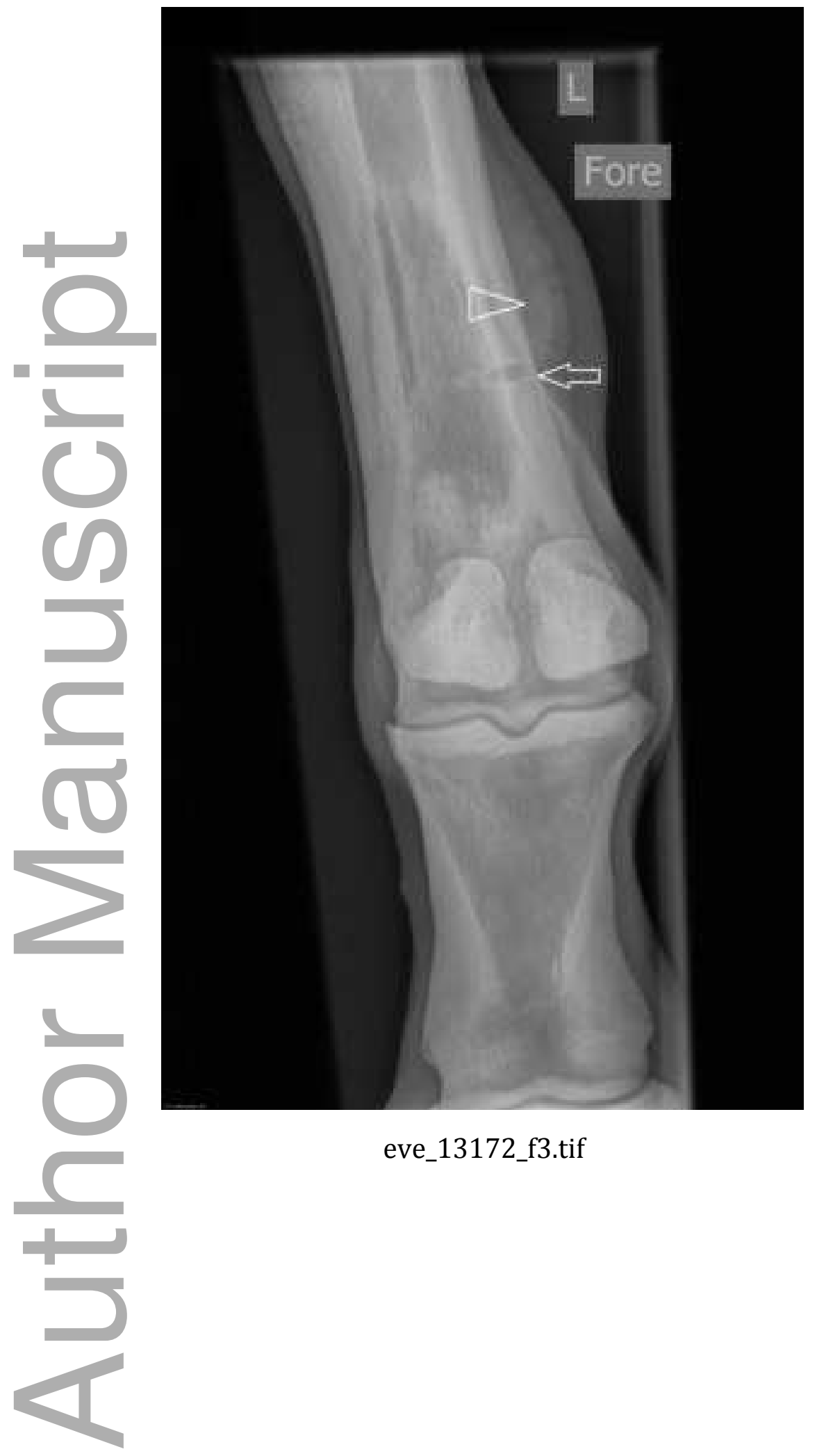

This article is protected by copyright. All rights reserved 


\section{University Library}

\section{- M M I N E R VA A gateway to Melbourne's research publications}

Minerva Access is the Institutional Repository of The University of Melbourne

Author/s:

Slack-Smith, V;Davies, HMS;Hilbert, BJ

Title:

Long bone failure after intraosseous regional perfusion

Date:

2021-03-01

Citation:

Slack-Smith, V., Davies, H. M. S. \& Hilbert, B. J. (2021). Long bone failure after intraosseous regional perfusion. EQUINE VETERINARY EDUCATION, 33 (3), pp.E30-E32. https:// doi.org/10.1111/eve.13172.

Persistent Link:

http://hdl.handle.net/11343/286376 